\title{
Influence of Emulsifiers on the Crystallization of Solid Lipid Nanoparticles
}

\author{
H. Bunjes, M.H.J. Koch ${ }^{1}$, K. Westesent \\ Friedrich Schiller Universiy, Institute of Pharmacy, Department of Pharmaceutical Technology, Lessingstrasse \\ 8, 07743 Jena, Germany \\ ${ }^{1}$ European Molecular Biology Laboratory, Hamburg Outstation, EMBL c/o DESY, Notkestrasse 85, 22603 \\ Hamburg, Germany
}

Aqueous colloidal dispersions of solid lipids, e.g., triglycerides, are being investigated as potential drug delivery systems. Lipid nanosuspensions with small particles and narrow particle size distributions are preferably obtained by emulsification of the molten matrix lipid in a hot aqueous phase with adequate stabilizers and subsequent crystallization of the dispersed lipid matrix. A variety of different emulsifiers has been used for the preparation of solid lipid nanoparticle dispersions, including phospholipids, bile salts, poloxamers and other ionic and nonionic surfactants. The type of emulsifier used in the formulation may not only affect the physical stability of the dispersion and the range of possible administration routes but also influence the crystallization and polymorphic behavior of the nanoparticles. Crystallization temperature and polymorphic transitions are important parameters for the preparation of triglyceride nanoparticles. During preparation, the emulsified dispersion must be cooled below the critical crystallization temperature of the triglyceride (i.e., much below its melting temperature) to crystallize the nanoparticles. Polymorphic transitions are important during formation of the solid nanoparticles since transition into a more stable triglyceride polymorph is accompanied by a rearrangement of the triglyceride molecules and an increase in lattice density. The type of crystal polymorph obtained and the kinetics of the transitions may thus have consequences for dispersion stability and drug loading. Against this background, the effect of different emulsifiers, with special regard to those considered for use in parenteral formulations, on the properties of tripalmitin nanodispersions was investigated. Besides the effect on the crystallization temperature the time-course of polymorphic transitions of the dispersed triglyceride was a major point of interest.

At the time point of the experiments, all dispersions displayed an X-ray diffraction pattern characteristic of the stable $\beta$-modification. The processes upon crystallization and subsequent polymorphic transitions were monitored in time-resolved simultaneous small and wide angle measurements during cooling of the dispersions which had been previously heated above the melting point of the nanoparticles. The transition kinetics of the dispersed triglyceride differed considerably (Fig. 1). In most cases, the transformation into the stable $\beta$-form was not complete within the time-scale of the experiment but the samples reached a quasi-equilibrium. Dispersions containing ionic surfactants usually retained a higher amount of $\alpha$-modification than those with nonionic surfactants. For example, dispersions stabilized solely with sodium glycocholate remained completely in the $\alpha$-form whereas for the dispersion stabilized with Cremophor EL virtually no reflection of the $\alpha$-modification was observed upon crystallization. Sodium glycocholate stabilized dispersions displayed an extraordinarily high stability of the $\alpha$-form during recrystallization. According to the wide angle diffractograms, the dispersions remained in the $\alpha$-form during the entire experiment (> $45 \mathrm{~min}$. after the onset of crystallization) independently of the cooling conditions (slow cooling $\left(\sim 0.3^{\circ} \mathrm{C} / \mathrm{min}\right.$.) to 20 or $10^{\circ} \mathrm{C}$, or rapid $\left(>5^{\circ} \mathrm{C} / \mathrm{min}\right.$.) cooling to $\left.5^{\circ} \mathrm{C}\right)$ after melting of the nanoparticles. Even upon heating $\left(1^{\circ} \mathrm{C} / \mathrm{min}\right.$.) the $\alpha$-crystalline structure was retained in these nanoparticle dispersions up to $35^{\circ} \mathrm{C}$ and above. The intensity of the small angle reflection of freshly crystallized glycocholate-stabilized dispersions depended highly on the crystallization conditions. The small angle reflection of the slowly cooled dispersion was nearly as strong as that of the native $\beta$-crystalline system. In contrast, there was only a very weak small angle reflection in the dispersion rapidly cooled to $5^{\circ} \mathrm{C}$ although the intensity of its wide angle reflection was comparable to that of the slowly cooled dispersion. This unusual $\alpha$-form, which seems not to contain a highly ordered layered structure, transforms into the regular one upon slow heating. Electron microscopic investigations revealed that the differences in the X-ray patterns were accompanied by different particle morphologies. These results indicate that major rearrangements still may take place in solid lipid nanoparticles after recrystallization [1]. 

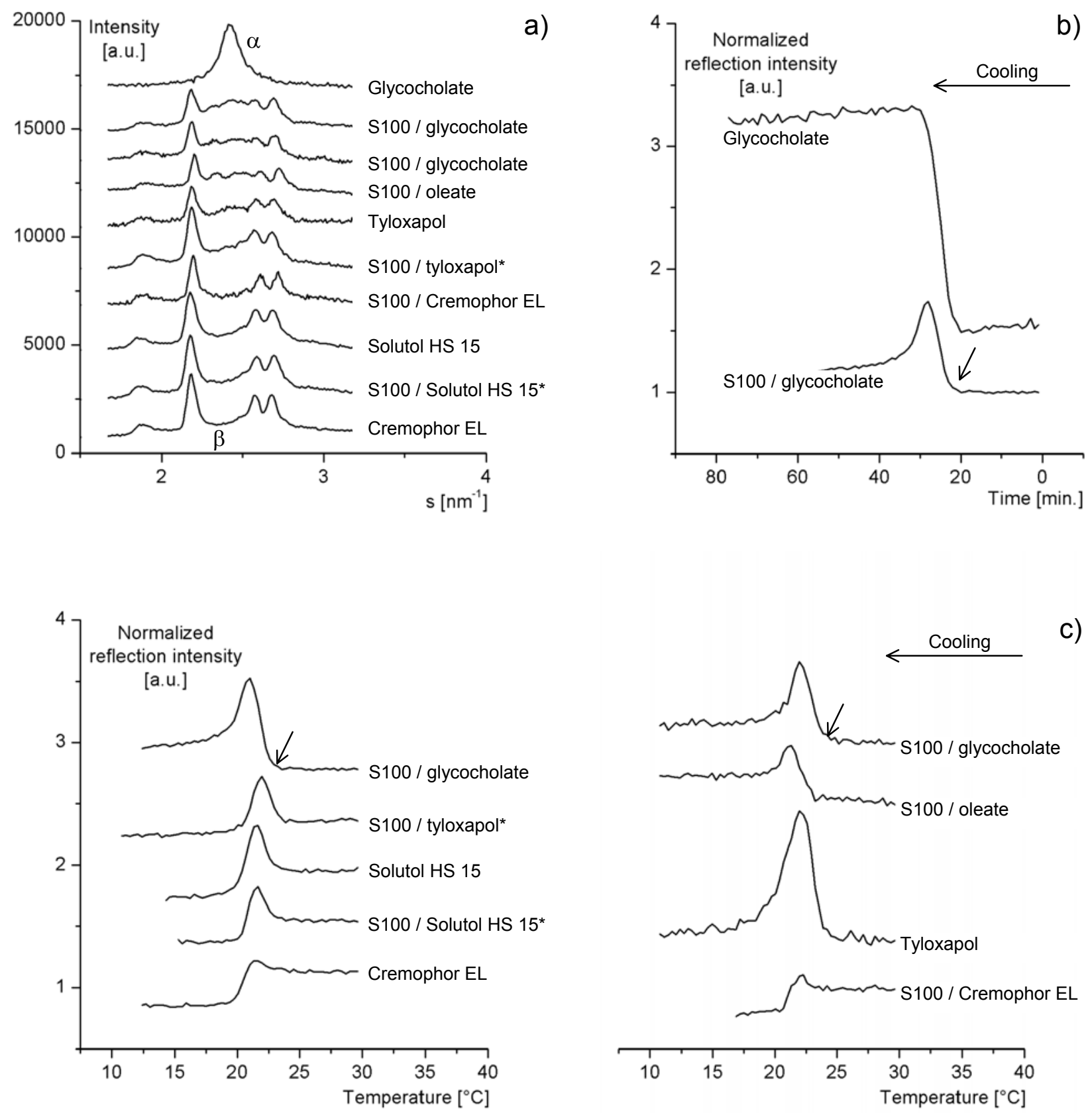

Figure 1: Wide-angle X-ray diffraction during recrystallization of tripalmitin nanoparticles stabilized with different surface active agents (S100: soybean phospholipid) upon cooling. (a) Wide-angle diffractograms of differently stabilized tripalmitin dispersions approximately $20 \mathrm{~min}$ after the onset of recrystallization (average of three 1-minute diffractograms). (b, c) Evolution of the X-ray scattering intensity of the dispersions in the region of the $\alpha$-reflection (around $0.415 \mathrm{~nm}$ ) versus time (b) or temperature (c). At high temperatures, the curves reflect only the diffuse scattering of the samples. The onset of crystallization of the nanoparticles is marked with an arrow for the phospholipid/glycocholate stabilized dispersions. In (b), the S100/glycocholate dispersion was cooled continuously, whereas the dispersion stabilized solely with glycocholate was kept isothermal after continuous cooling to $20^{\circ} \mathrm{C}$. Systems marked with an asterisk became semisolid during the cooling program.

\section{References}

[1] Bunjes H., Koch M.H.J., Westesen K., J. Pharm. Sci. 92, 1509 (2003). 